\title{
Multiple cranial nerve schwannomas and meningiomas as a hallmark sign of neurofibromatosis type 2 in a child
}

\author{
Ahmet Mesrur Halefoğlu \\ Department of Radiology, Sisli Hamidiye Etfal Training and Research Hospital 34360, Sisli, Istanbul, Turkey. \\ E-mail: halefoglu@hotmail.com \\ Received: 19th January 2017, Revised 21st February 2017, Accepted: 21st February 2017
}

SUMMARY: Halefoğlu AM. Multiple cranial nerve schwannomas and meningiomas as a hallmark sign of neurofibromatosis type 2 in a child. Turk J Pediatr 2018; 60: 107-110.

Neurofibromatosis type 2 is a rarely encountered autosomal dominant disorder manifesting with typical radiological findings. These patients have a predilection for development of benign tumors in the central nervous system. Although the presenting symptom is most commonly hearing loss due to acoustic schwannomas, symptoms emanating from other cranial tumors are not uncommon. Herein, we described a 16-year-old male patient presented with multiple meningiomas and cranial nerve schwannomas revealed by magnetic resonance imaging. He fulfilled the diagnostic criteria of neurofibromatosis type 2 and underwent treatment. We emphasized the role of radiology in the early diagnosis of this inherited disorder in order to provide a better prognosis.

Key words: magnetic resonance imaging, neurofibromatosis type 2, children.

Neurofibromatosis type 2 (NF 2), first described in 1822 by the Scottish surgeon Wishart, is a rare inherited autosomal disorder and represents with multiple benign cranial and spinal tumors, peripheral neuropathy, opthalmological and cutaneous lesions. ${ }^{1,2}$ The disease is encountered in one of 25,000 live births. ${ }^{1}$ Eighteen percent of NF 2 patients manifest with the symptoms of disease at the age of 15 years or less. ${ }^{3} \mathrm{NF}$ 2 is caused by a mutation on chromosome $22 .{ }^{4}$ Bilateral vestibular schwannomas are the classic hallmark of NF 2, which predisposes patients to develop multiple schwannomas on cranial, spinal and peripheral nerves and to intracranial and intraspinal meningiomas and intramedullary ependymomas.5,6 Although most of these mentioned central nervous system tumors have a predilection for slow growing, sometimes they may show an aggressive growth pattern especially in children which leads to a poor prognosis. ${ }^{7}$ In this case report, we described magnetic resonance imaging (MRI) findings of multiple cranial tumors in a 16-year-old male patient presenting with NF 2 and highlighted the importance of radiological imaging in the diagnosis of this rare disorder.

\section{Case Report}

A 16-year-old male was admitted to the neurology department complaining of chronic headache, left-sided tinnitus, vision deficit in the left eye and weakness in his upper limbs. There was no significant family history. Neurological examination revealed bilateral Babinski‘s reflex, right-sided paresis and mild muscular atrophy and right hypoglossal nerve paresis with deflection of the tongue to the left. An audiogram was performed and showed moderate left sensorineural hearing loss. Following neurological examination the patient was referred to brain MRI for further evaluation using a 1.5 tesla magnet (Avanto- SQ Engine; Siemens, Erlangen, Germany). During this examination, axial and sagittal T1 weighted, axial and coronal T2 weighted, axial and coronal FSE IR (flair), axial SWI (susceptibility weighted) and finally following intravenous gadolinium DTPA administration $(0.1 \mathrm{mmol} /$ $\mathrm{kg}$ ) axial and coronal post-contrast $\mathrm{T} 1$ weighted images were obtained. These images revealed homogenously intense enhancing masses along the cranial nerves including a large left cerebellopontine angle cisternal mass along the 8th cranial nerve consistent with acoustic 


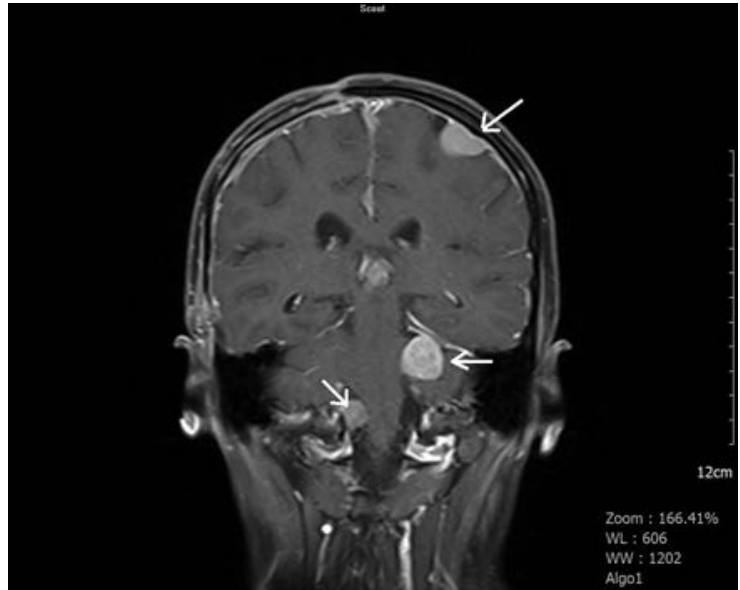

Fig. 1. Coronal SE T 1 weighted post-contrast image, a right hypoglossal and a left acoustic nerve contrast-enhancing masses consistent with schwannomas are seen. A left parietal extra-axial and third ventricular homogenously enhancing masses indicating meningiomas are also detected.

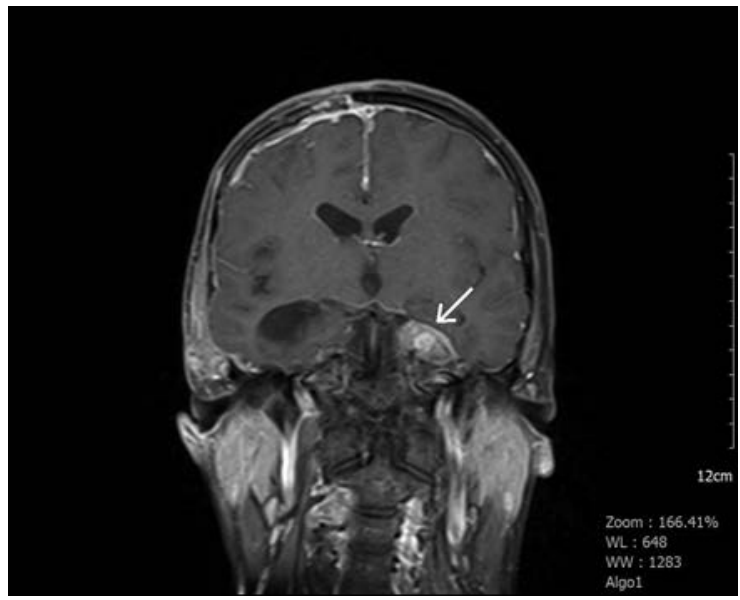

Fig. 2. Coronal SE T 1 weighted post-contrast image clearly depicts an intensely enhancing mass in the left Meckel cave region consistent with a left trigeminal schwannoma.

schwannoma and in the region of medulla oblangata along the right 12 th cranial nerve consistent with hypoglossal schwannoma (Fig. 1). There was also another similar mass lesion in the left Meckel cave region, where the Gasserian ganglion was located in, indicating left trigeminal schwannoma (Fig. 2). Besides multiple cranial nerve involvement, along the left optic nerve sheath a homogenously enhancing mass typical of meningioma was detected (Fig. 3). Finally, both infra- and supratentorial extra-axial regions and also in the third ventricular area, characteristically enhancing multiple meningiomas were discovered (Fig. $1,3,4)$. He also underwent whole spinal contrast-enhanced MRI in order to rule out

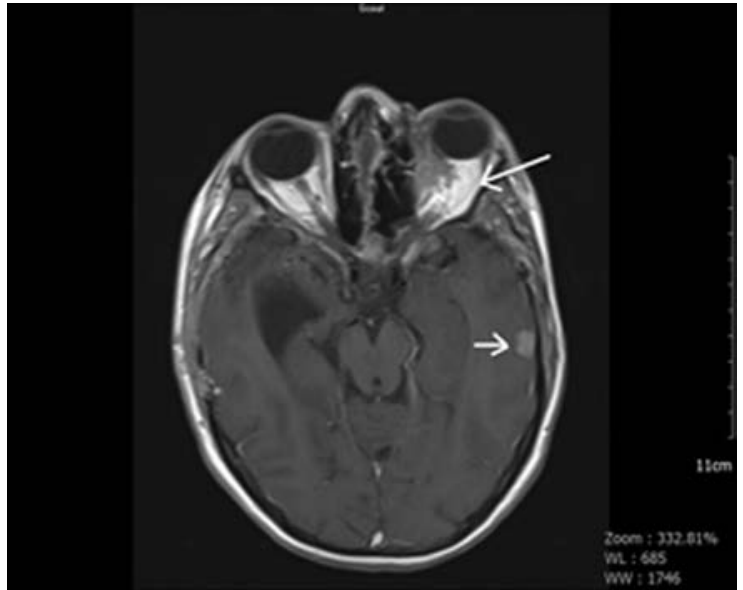

Fig. 3. Axial SE T 1 weighted post-contrast image, a homogenously enhancing mass along the left optic nerve region is seen. Left optic nerve meningioma and also a small left temporal meningioma were found.

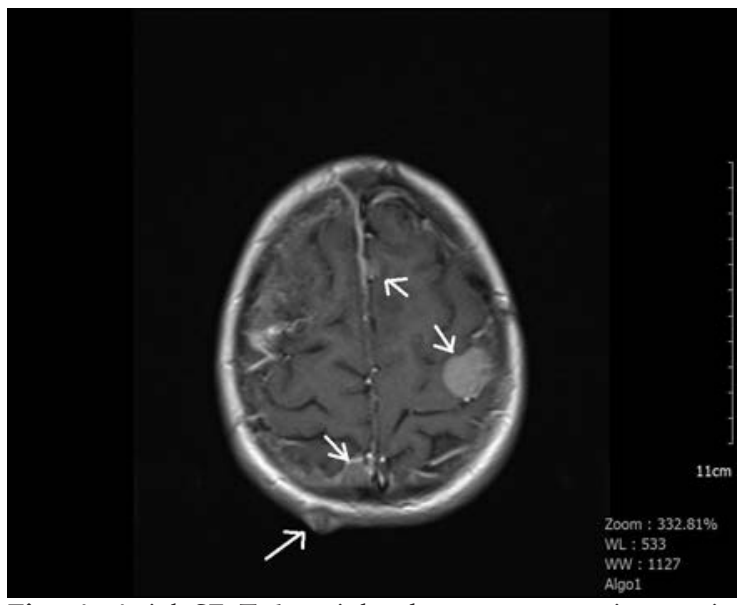

Fig. 4. Axial SE T 1 weighted post-contrast image, in the convexity level, a relatively huge contrast enhancing meningioma is seen in the left posterior frontal region. Also a smaller similar one can be seen in the right posterior parietal region near the falx cerebri.

possible spinal tumors which was negative. A combination of multiple cranial nerve schwannomas and multiple meningiomas, the patient was thought to have NF type 2 disorder. He was administered symptomatic medical treatment and referred to neurosurgery department for further investigation including genetic analysis and possible surgical procedures.

Informed written consent of the patient was obtained for publication

\section{Discussion}

Definite NF 2 is present in an individual who has bilateral vestibular nerve schwannomas 
or in an individual who has a first-degree relative with NF 2, is younger than 30 years old age, and presents with unilateral vestibular nerve schwannoma or two of the following: meningioma, glioma, schwannoma, juvenile posterior subcapsular lenticular opacities, and juvenile cortical cataracts. ${ }^{8}$ The term MISME has been proposed to the NF 2 syndrome, due to multiple inherited schwannomas (MIS), meningiomas (M), and ependymomas (E). In about half of the patients, a family history is not present and therefore a new spontaneous mutation causes the disease. ${ }^{9}$ The birth incidence is significantly higher than the diagnostic prevalence because many cases do not develop features of the disease until the third decade or later, and many other cases die before this time. The mean survival rate of the disease after diagnosis is approximately 15 years. ${ }^{10}$ The majority of patients with NF 2 present with hearing loss, which is usually unilateral at onset. In our case report, left-sided moderate degree sensorineural hearing loss was found at audiogram and the patient was complaining of tinnitus on the left. A significant proportion of cases (20$30 \%)$ present with an intracranial meningioma, spinal tumor or cutaneous tumor. ${ }^{10}$ Ophthalmic involvement is also common in NF 2 cases. Between $60 \%$ to $80 \%$ of NF 2 patients have cataracts, which are usually presenile posterior subcapsular lenticular opacities that rarely require removal. ${ }^{11}$ Optic nerve meningiomas can cause visual loss in the first years of life and extensive retinal hamartomas can also affect vision. ${ }^{12}$ We revealed an optic nerve sheath meningioma in our NF 2 patient which led to vision loss on the left. The skin is a useful aid to diagnosis, but cutaneous features in NF 2 are much more subtle than in NF 1. About $70 \%$ of NF 2 patients have skin tumors, but only $10 \%$ have more than 10 skin tumors. ${ }^{10}$ decision MRI with gadolinium contrast enhancement is regarded as gold standard in terms of a precise diagnostic decision. Especially in the search of acoustic tumors, MRI is highly sensitive than the other modalities. Computed tomography (CT) has a poor sensitivity to detect small vestibular schwannomas and hence has been largely replaced by MRI. We also preferred MRI examination in our patient in terms of revealing multiple probable intracranial tumors. MRI is able to detect tumors as small as 1-2 $\mathrm{mm}$ in diameter on cranial and spinal nerve roots. The classic diagnostic hallmark of NF is bilateral vestibular schwannomas and is seen more than $90 \%$ of patients. Most of these schwannomas arise from the vestibular part of the 8th cranial nerve in the cerebellopontine angle cistern and approximately one-third from the spinal nerve roots. ${ }^{13}$ The predilection for the superior vestibular branch of the 8th cranial nerve is not yet explained in these cases. It is reported that more than $99 \%$ of vestibular schwannomas in NF 2 are benign, but they remain an important cause of mortality due to their location. ${ }^{1}$ Another common finding on MRI is schwannomas involving other cranial nerves. These occur most commonly on the 5th nerve, but every cranial nerve can be affected by the disease. ${ }^{14}$ But these schwannomas in contrast to vestibular ones, usually do not grow to a size that necessitate surgical excision. Our patient had a huge left vestibular schwannoma and also demonstrated a left trigeminal and a right hypoglossal schwannomas as well. The other type tumor in NF 2 cases is meningioma. These can easily be detected on MRI as enhanced areas on meninges around the spinal cord, brain or optic nerves. Multiple homogenously enhancing supra and infratentorial meningiomas including a left optic nerve sheath one, were present in our NF 2 patient.

Mautner et al. ${ }^{15}$ in their study showed 48 NF 2 patients who presented with vestibular schwannomas in $46(96 \%)$, spinal tumors in $43(90 \%)$, posterior subcapsular cataracts in $30(63 \%)$, meningiomas in $28(58 \%)$ and trigeminal schwannomas in $14(29 \%)$. Wayman et al. ${ }^{16}$ reported 11 patients who have NF 2 disease and in this series, all patients had acoustic neuromas, 6 patients had meningiomas and 8 patients demonstrated other cranial nerve tumors. Nunes et al. ${ }^{17}$ published clinical data of $12 \mathrm{NF} 2$ patients who were younger than 18 years old and in large number of patients with positive family history. Radiological examinations revealed cranial meningiomas in $75 \%$ of cases, cranial schwannomas in $83 \%$ of cases and spinal cord tumors in $75 \%$ of cases. Bosch et al. ${ }^{18}$ reported that children with early onset of disease presented with ophthalmologic symptoms however, 8th cranial nerve impairment was detected in children who had late disease onset. In NF type 2 patients, 
hearing loss is often accompanied by tinnitus, occurring in around $60 \%$ of adults and up to $30 \%$ of children. ${ }^{19}$ Intracranial meningiomas appear in $45-58 \%$ of patients with NF 2 and although most of them are benign in nature, they may cause clinical symptoms related to their size and location. It has been proven that meningiomas related with NF 2 disorder show frequently higher proliferation activity and have a tendency to form more atypical and anaplastic grades than sporadic meningiomas. ${ }^{20}$ In our NF 2 patient, 5th, 8th and 12th nerve acoustic schwannomas and multiple meningiomas including left optic nerve sheath were detected.

Surgical treatment of patients with NF 2 is complex and patients optimally should be managed by a multidisciplinary team. The typical treatment strategy for patients with vestibular schwannomas is 'watch and wait then rescan', complete surgical resection and stereotactic radiotherapy. The authors reported that more than $50 \%$ of vestibular schwannomas were stable in size, therefore 'watch and wait then rescan' protocol could be applied in the proper course of the disease. ${ }^{7}$ The role of stereotactic radiotherapy is not yet clearly established. ${ }^{7}$ On the other hand, the majority of meningiomas can be safely and radically resected in NF 2 patients.

As a conclusion, presentation of NF 2 patients with multiple intracranial tumors leads to a poor prognosis. MRI plays a crucial role in the diagnosis of the disease. In order to achieve a better treatment in these patients unification of the management strategies is highly desirable.

\section{REFERENCES}

1. Asthagiri AR, Parry DM, Butman JA, et al. Neurofibromatosis type 2. Lancet 2009; 373: 19741986.

2. Mautner VF, Baser ME, Thakkar SD, Feigen UM, Freidman JM, Kluwe L. Vestibular schwannoma growth in patients with neurofibromatosis type 2: A longitudinal study. J Neurosurg 2002; 96: 223-228.

3. Janse AJ, Tan WF, Majoie ChBLM, Bijlsma EK. Neurofibromatosis type 2 diagnosed in the absence of vestibular schwannomas. A case report and guidelines for a screening protocol for children at risk. Eur J Pediatr 2001; 160: 439-443.

4. Rouleau GA, Wertelecki W, Haines JL, et al. Genetic linkage of bilateral acoustic neurofibromatosis to a DNA marker on chromosome 22. Nature 1987; 329: 246-248.
5. Egelhoff JC, Bates DJ, Ross JS, Rothner AD, Cohen $\mathrm{BH}$. Spinal MR findings in neurofibromatosis types 1 and 2. AJNR Am J Neuroradiol 1992; 13: 1071-1077.

6. Mautner VF, Tatagiba M, Lindenau M, et al. Spinal tumors in patients with neurofibromatosis type 2: MR imaging study of frequency, multiplicity and variety. AJR Am J Roentgenol 1995; 165: 951-955.

7. Moffat DA, Quaranta N, Baguley DM, Hardy DG, Chang P. Management strategies in neurofibromatosis type 2. Eur Arch Otohinolaryngol 2003; 260: 12-18.

8. Gutmann DH, Aylsworth A, Carey JC, et al. The diagnostic evaluation and multidisciplinary management of neurofibromatosis 1 and neurofibromatosis 2. JAMA 1997; 278: 51-57.

9. Evans DG, Mason S, Huson SM, Ponder M, Harding AE, Strachan T. Spinal and cutaneous schwannomatosis is a variant form of type 2 neurofibromatosis: a clinical and molecular study. J Neurol Neurosurg Psychiatry 1997; 62: 361-366.

10. Evans DG, Huson SM, Donnai D, et al. A clinical study of type 2 neurofibromatosis. Q J Med 1992; 84: 603-618.

11. Ragge NK, Baser ME, Klein J, et al. Ocular abnormalities in neurofibromatosis 2. Am J Ophtalmol 1995; 120: 634-641.

12. Bouzas EA, Parry DM, Eldridge R, Kaiser-Kupfer MI Visual impairment in patients with neurofibromatosis 2. Neurology 1993; 43: 622-623.

13. Russell DS, Rubinstein LJ. Tumors of the cranial, spinal peripheral nerve sheaths. In: Russell DS, Rubinstein LJ (eds). Pathology of the Tumours of the Nervous System (5th ed). London: Edward Arnold; 1989: 533589.

14. Mautner VF, Lindenau M, Baser ME, et al. The neuroimaging and clinical spectrum of neurofibromatosis 2. Neurosurgery 1996; 38: 880-885.

15. Mautner VF, Tatagiba M, Laute S, Guthoff R, Samii M, Pulst SM. Clinical and genetic analysis of neurofibromatosis type 2 (abstr). Neurology 1992; 42(Suppl 3): 473.

16. Wayman JW, Dutcher PO Jr, Manzione JV, Nelson CN, Kido DK. Gadolinium-DTPA-enhanced magnetic resonance scanning in cerebellopontine angle tumors. Laryngoscope 1989; 99: 1167-1170.

17. Nunes F, MacCollin M. Neurofibromatosis 2 in the pediatric population. J Child Neurol 2003; 18: 718-724.

18. Bosch MM, Boltshauser E, Harpes P, Landau K Ophtalmogic findings and long- term course in patients with neurofibromatosis type 2. Am J Ophtalmol 2006; 141: 1068-1077.

19. Ruggieri M, Iannetti P, Polizzi A, et al. Earliest clinica manifestations and natural history of neurofibromatosis type 2 (NF2) in childhood: a study of 24 patients. Neuropediatrics 2005; 36: 21-34.

20. Perry A, Giannini C, Raghawan R, et al. Aggressive phenotypic and genotypic features in pediatric and NF2-associated meningomas: a clinicopathologic study of 53 cases. J Neuropathol Exp Neurol 2001; 60: $994-$ 1003. 